\title{
Application Analysis of Swietenia Mahagoni (Mahogany) Seed's Unique Aerodynamical Landing Characteristics
}

\author{
Debashis Baidya \\ Mathematics Discipline, Khulna University, Khulna, Bangladesh \\ Email address: \\ bddebashis@outlook.com \\ To cite this article: \\ Debashis Baidya. Application Analysis of Swietenia Mahagoni (Mahogany) Seed's Unique Aerodynamical Landing Characteristics. \\ American Journal of Aerospace Engineering. Vol. 3, No. 3, 2016, pp. 31-35. doi: 10.11648/j.ajae.20160303.12
}

Received: October 30, 2016; Accepted: November 14, 2016; Published: November 25, 2016

\begin{abstract}
A model of safe landing capsule and ICBM multi war head transportation system for cluster type bomb attack by analysis of seed landing trajectory. Swietenia mahagoni (mahogany) seed has the uniqueness on its falling characteristics. At the time of falling, mahogany seed rotates in the axis center which is similar to the gyration of propeller towards its pivot point. The seeds look and behave like helicopter rotors. The gyration slows down the landing time whereby it is furthering the landing distance of the mahogany seeds. In this paper focused on this characteristic of the mahogany seed for real world application analysis and also the whole seed model application analysis also including some tests on the seeds.
\end{abstract}

Keywords: Aerodyanmics of Falling Particle, Landing System, Aerodynamic Landing, War Head, ICBM, Aerodynamics, Terminal Velocity, Swietenia Mahagoni

\section{Introduction}

Helicopters (also called Whirlybirds) include seeds or oneseeded fruits (samaras) with a rigid or membranous wing at one end. The wing typically has a slight pitch (like a propeller or fan blade), causing the seed to spin as it falls. Depending on the wind velocity and distance above the ground, helicopter seeds can be carried considerable distances away from the parent plant. The spinning action is similar to auto-rotation in helicopters, when a helicopter "slowly" descends after a power loss. It is an observed fact that not all falling objects travel straight downward under the influence of gravity. Leaves, paper and seeds all follow complicated trajectories as they fall. This certain phenomenon is greatly utilized by nature; one good example can be seen in trees in their seed dispersal. Seed dispersal is an important process in plant population dynamics. There are a lot of tree species among them are Swetenia mahogany that have developed rather special way of dispersing seeds in the environment. One of the trees that exhibit this seed dispersal behavior, Swetenia mahogany, can be found around Khulna University Campus. In this paper the Swetenia mahogany seed is used for it's aerodynamical characteristics, though there are many other seeds with this characteristics here mahogany seed is used for it's shape and simple structure. It is easier to use this simple structured but complex falling characteristic model for real life applications.

Nowadays parasuit is used for life saving while plane accident or air strike but there are too much limitations for use. As example it needs proper eject situation for pilot to eject, proper altitude for properly bloom and also a heavy hit while landing. So it can be very helpful if any landing capsule can be used which will be eject situation independent and will land safely. Here the falling trajectory characteristics of mahogany seed is useful.

Also nowadays modern ICBM carries multiple war heads and cluster missiles carries multiple explosives for wide ranged attack. Here a natural model and phenomena is analyzed for develop this technology for which the aerodynamical characteristics of mahogany seed may be useful.

\section{Structure and Seed Model Properties Analysis}

Mahogany seed is basically constructed by three element outer pod, inner pod and the seed. Outer pod is the seeds container which keeps the seeds covered and the inner pod contains the thin blade shaped seeds cluster. Here a possibly clear figures are provided below: 




Figure 1. Seed structure.

An experimental investigation of near field aerodynamics of wind dispersed rotary seeds has been performed using stereoscopic digital particle image velocimetry (DPIV). The detailed three-dimensional flow structure of the leading-edge vortex (LEV) of autorotating mahogany seeds (Swietenia macrophylla) in a low-speed vertical wind tunnel is revealed by E. Salcedo, C. Treviño, R. O. Vargas, L. MartínezSuástegui (2013 216: 2017-2030; doi: 10.1242/jeb.085407).

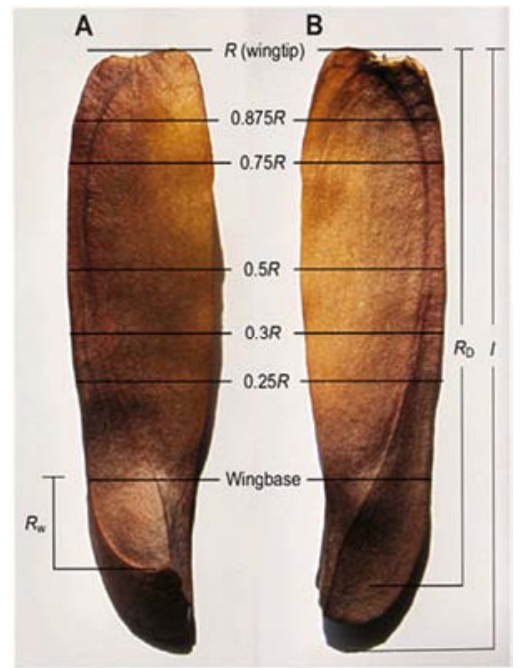

Figure 2. Spanwise positions along the wing for flow measurement, Here $R$ is the distance from the wingbase to wingtip, $R w$ is the disance from the seed's center of rotation to the wingbase, $R d$ is the distance from the seed's center of rotation to the wingtip.

Based on the measured flow patterns, the following conclusions drawn by E. Salcedo, C. Treviño, R. O. Vargas, L. Martínez-Suástegui (2013 216: 2017-2030; doi: 10.1242/jeb.085407):

a) No LEV is present at the wingbase.

b) At $0.25 R$, the flow separates at the LE, a region of positive (clockwise) vorticity is generated, the LEV appears above the airfoil, and the separated flow reattaches close to the TE.

c) The LEV increases in size and strength while spiraling outward towards the wingtip with a straight cone pattern, which is responsible for enhancing lift generation. The strong flow strain in the spanwise direction stretches the LEV, thus increasing its intensity and promoting the attachment of the latter against the airfoil. d) The LEV core and spanwise velocity reach a plateau at $0.75 R$ and decrease in size and strength at $0.875 R$.

e) In a spanwise region between $0.9 R$ and the wingtip, the $\mathrm{LEV}$ is reoriented into the tip vortex.

\section{Falling Phase Analysis and Trajectories}

Now some falling behavior is observed by letting the seed fall from different phase. Here the falling phase, landing phase and terminal velocity are observed and measured for figuring the falling trajectory and landing phase of the seed. It is observed that after dropping the seed in any phase it falls a short period freely by gravitational force and then it gains it's unique aerodynamical falling characteristics and falling trajectories. The free falling period differ on various falling phase.

\subsection{Phase 1: When Dropped Parallely}

When the seed is dropped paralley to ground then it falls a freely for a period which is defined as free falling and then it gets the unique aerodynamical falling characteristics. We get the falling trajectory graph as below when dropped parallely:

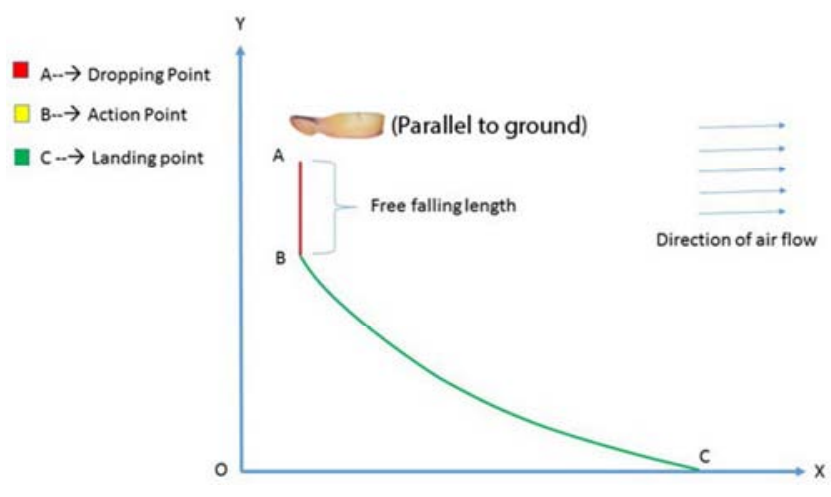

Figure 3. When dropped parallely.

In Phase 1: the seed falls parallely with ground from the point A (Dropping point) and it fall freely since the point B so the length or height $\mathrm{AB}$ is the free falling distance of the seed while fall parallely. At point B it automatically get balanced and gains the unique aerodynamical velocity and falling characteristics so here the point B is called the action point. After getting the unique aerodynamical characteristics the seed moves through a parabolic trajectory and lands at the point $\mathrm{C}$ (Landing point).

Here it is noticed that at this falling phase it has a standard free falling length and trajectory is normal.

\subsection{Phase 2: Wingbase Down}

When the seed is dropped with wingbase down side then it falls freely for a period and then it gets the unique aerodynamical falling characteristics. We get the falling trajectory graph as below when dropped keeping wingbase down side: 


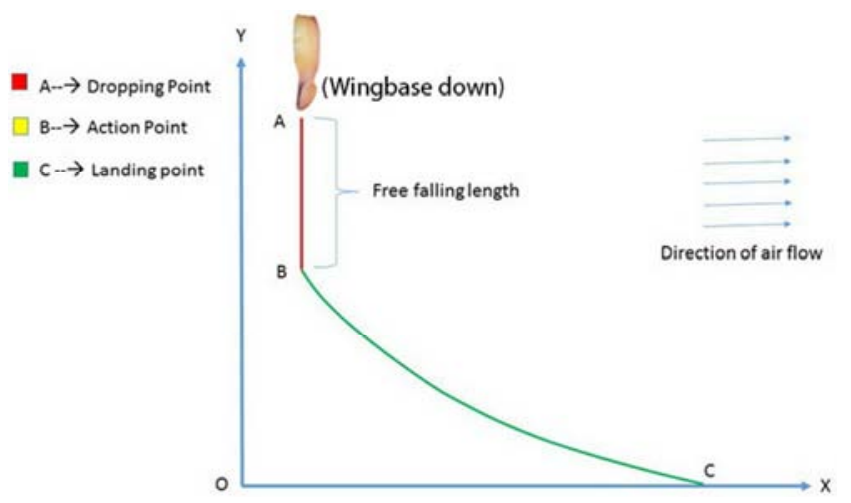

Figure 4. When dropped wingbase down (maximum free falling length).

Here it is noticed that the free falling length $\mathrm{AB}$ is greater than phase 1 and also the trajectory $\mathrm{BC}$ is shorter than phase 1 .

\subsection{Phase 3: Wingbase Is Vertical to Ground \& Seed Is Horizontal}

When the seed is dropped keeping the wingbase vertically and the whole seed horizontally to ground then it also falls freely for a period which is defined as free falling and then it gets the unique aerodynamical falling characteristics. We get the falling trajectory graph as below when dropped parallely:

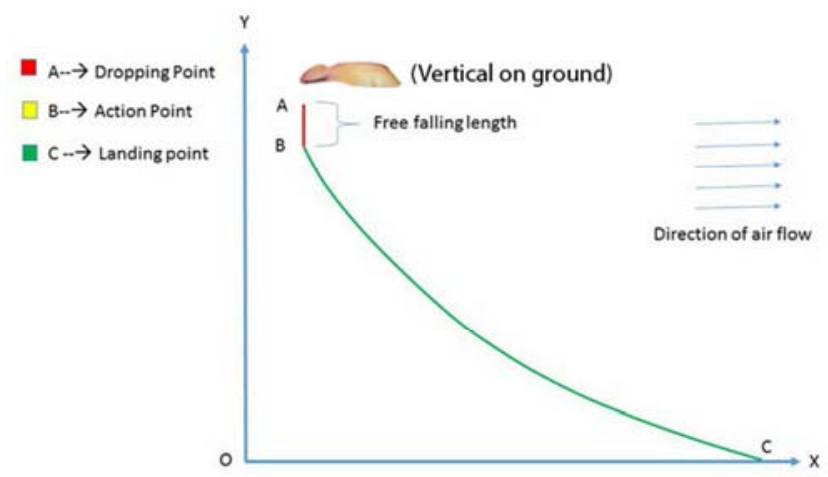

Figure 5. When dropped parallely but wingbase vertically (minimum free falling length).

Here in this phase noticed that the free falling length is much more shorter than Phase 1 and Phase 2 and also the action trajectory $(\mathrm{BC})$ is more lengthy.

\subsection{Phase 4: Throw Upward Against Gravity and Airflow}

Now When the seed thrown upward to sky against gravity and airflow direction (In Fig. 4 Thrown from point A in). Then it falls differently than other phases. At this phase as soon as the seed start falling it immediately start rotation and gets the unique characteristics, at this phase there is no free falling period or free falling length. It directly gets uniqueness from point B (Where it starts falling).

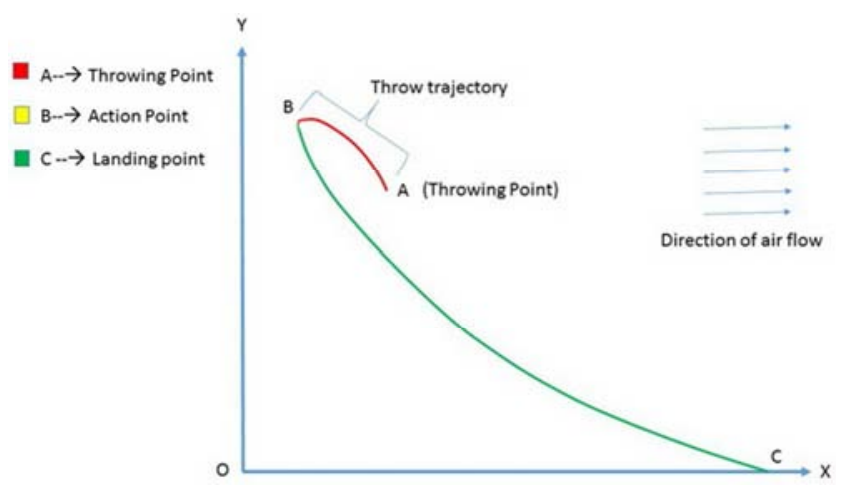

Figure 6. When throwing upward (no free falling distance).

Therefore the Action point and the Falling point is same. Note: Here we get no free falling distance.

\subsection{Phase 5: Throw Upward Against Gravity and Airflow}

Now When the seed thrown upward to sky against gravity and toward airflow direction (In Fig. 4 Thrown from point $\mathrm{A}$ in). At this phase as soon as the seed start falling it immediately start rotation and gets the unique characteristics, So at this phase there is no free falling period.

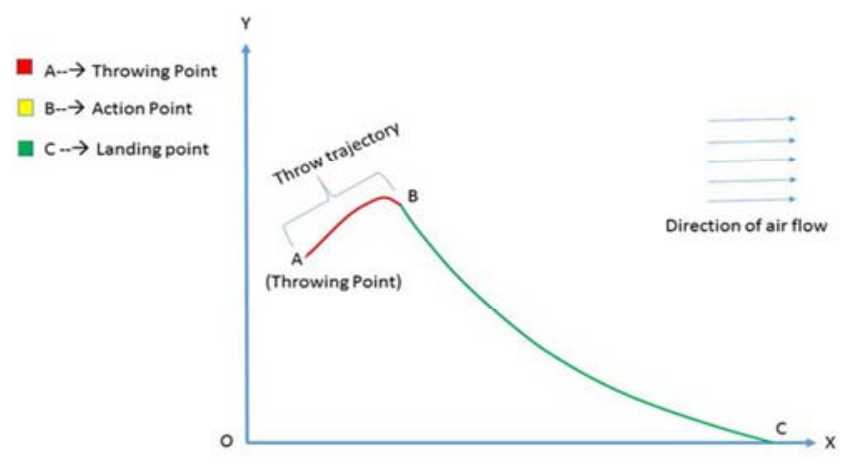

Figure 7. When thrown upward at air flow direction.

It directly gets uniqueness from point B (Where it starts falling). Therefore the Action point and the Falling point is same.

Note: Here we get no free falling distance.

\subsection{Overall Falling or Landing Trajectory}

Now after examining the phases we noticed some complex properties and now some discussion is made.

a) When falling with wingbase is vertical to ground \& seed is horizontal then the free falling length is minimum and action trajectory is longer but not maximum.

b) When it is thrown against gravity then there is no free falling length and trajectory is maximum.

c) The trajectory is always parabolic. 


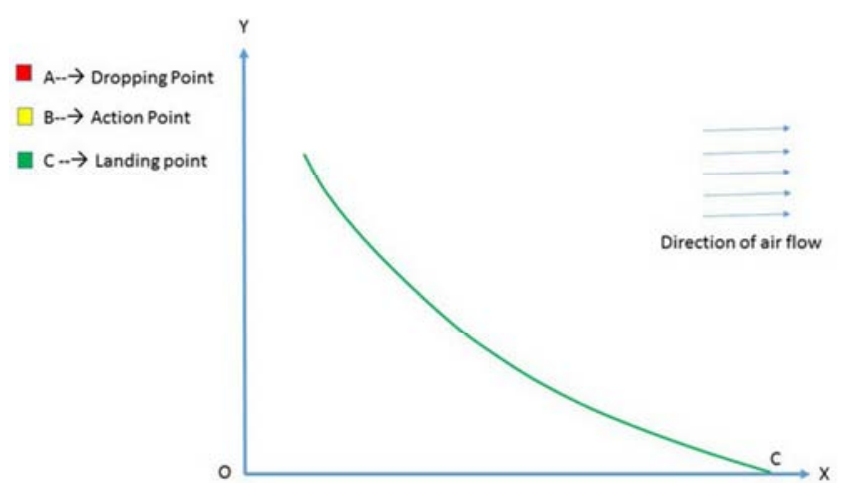

Figure 8. General trajectory of the seed.

\section{Total Thrust and Aerodynamic Performance Measurement}

The geometry and kinematics of rotary seeds have been described previously (Azuma and Yasuda, 1989; Greene and Johnson, 1990; Minami and Azuma, 2003; Yasuda and Azuma, 1997).

The seed reach at ground at point $\mathrm{C}$ and though this research is for using this characteristic for developing a safe landing capsule so we need to know and measure the terminal velocity, Thrust etc, which we can get from the research paper by E. Salcedo, C. Treviño, R. O. Vargas, L. Martínez-Suástegui, Journal of Experimental Biology 2013 216: 2017-2030; doi: 10.1242/jeb.085407

where $\rho$ is fluid density, CL is the local lift coefficient. The total thrust is then [Ref: E. Salcedo, C. Treviño, R. O. Vargas, L. Martínez-Suástegui ]:

$$
T=\cos \gamma \frac{\rho}{2} U_{\infty}^{2} R_{D} \gamma \int_{\varepsilon}^{1} C_{L} C \xi \sqrt{1+\lambda^{2} \xi^{2}}\left(1+\frac{1}{\lambda^{2} \xi^{2}}\right) d \xi
$$

where $\varepsilon=R_{W} / R_{D}$, with $R_{W}$ being the distance from the seed's center of rotation to the wingbase.

$$
C_{T}=\frac{2 M g}{\rho \bar{C} R_{D} U_{\infty}^{2}}
$$

Where $\mathrm{C}_{\mathrm{T}}$ Is thrust coefficient. $\mathrm{Mg}$ is the weight of the seed, $\xi=r / R_{D}$ Then, the definition of the thrust coefficient involves only the descending velocity. Once the thrust coefficient is known, the lift coefficient can be obtained.

\section{Final Model}

The vision of this research paper is to obtain a unique aerodynamical model to make it's application in our real world for a significant change, and so after analyzing the characteristics and properties of a Swietenia mahagoni seed two model is formed for use in our real world.

\subsection{Safe Landing Capsule}

For using as a alternative of parasuit. Life saving kit for pilots specially fighter planes and air travelers.



Figure 9. Proposed capsule model.

\subsection{Cluster ICBM}

An improved and revised model for ICBM (intercontinental ballistic missile) for cluster warhead transport and wide range attacks.



Figure 10. Proposed ICBM Model (Followed by the natural seed model).

This model is identical of full mahogany seed with inner and outer pod. Here the safety capsules (like the seeds) can be used like cluster warheads which can spread and cover more attack area by it's unique aerodynamic characteristics.

\section{Result \& Discussion}

\subsection{Safety Capsule}

a) How can be used: A flying capsule can be made like the seed for using it as a better alternative of parasuit and as a life saving device while air travel. Metallic capsule like this shape can be made and use it life saving or emergency saving capsule.

b) Why use: It should use cause it is better than parasuit and other life saving kit while air travel.

c) Advantages: It's better cause it can be trigger for use at any situation which means no need to wait a pilot for proper situation to eject cause it can be trigger at any 
angle and any time and will land safely.

\subsection{Cluster ICBM}

a) How can be used: In this ICBM model warheads are made like the safety capsule or like the seed so that it get the mahogany seed's properties and are stacked at the side of the missile rather than head but also the head section can also carry the warhead.

b) Why use: This ICBM model is much more effective cause when attack it will trigger the capsule warheads just like the seed dispersion and the warheads will cover more attack area with it's own trajectory. It will be much more pay load capable and able for cluster type attack.

c) Advantages: More attack are coverage, able to load more payload, cluster type attack with ICBM.

\section{Conclusions}

Still now plane accident is very much dangerous and cost so much lives, so this type of air safety capsule can save a lot of life and can make air travel much more safer. Also global politics are not much acceptable, so for our national security we need superior weapons day by day. This cluster ICBM is definitely very effective also at less cost. The analysis of this research paper easily shows a very natural model in our real world.

\section{Acknowledgments}

This paper work was my thinking and interest but this was never possible without the help of my dearest Bonna Biswas. Her inspiration and support is the only cause of successfully finishing this paper. I always wish her smile forever.

\section{References}

[1] E. Salcedo, C. Treviño, R. O. Vargas, L. Martínez-Suástegui. Stereoscopic particle image velocimetry measurements of the three-dimensional flow field of a descending autorotating mahogany seed (Swietenia macrophylla). Journal of Experimental Biology 2013 216: 2017-2030; doi: 10.1242/jeb.085407.

[2] Ansari, S. A., Phillips, N., Stabler, G., Wilkins, P. C., Żbikowski, R. and Knowles, K. (2009). Experimental investigation of some aspects of insect-like flapping flight aerodynamics for application to micro air vehicles. Exp. Fluids 46, 777-798.

[3] Azuma, A. and Yasuda, K. (1989). Flight performance of rotary seeds. J. Theor. Biol. 138, 23-54.

[4] Dickinson, M. H., Lehmann, F.-O. and Sane, S. P. (1999). Wing rotation and the aerodynamic basis of insect flight. Science 284, 1954-1960.

[5] Ellington, C. P. (1984). The aerodynamics of hovering insect flight. III. Kinematics. Philos. Trans. R. Soc. B 305, 41-78.

[6] Ellington, C. P., Berg, C. V. d., Willmott, A. P. and Thomas, A. L. R. (1996). Leading-edge vortices in insect flight. Nature 384, 626-630.

[7] Greene, D. F. and Johnson, E. A. (1990). The aerodynamics of plumed seeds. Funct. Ecol. 4, 117-125.

[8] Howe, H. F. and Smallwood, J. (1982). Ecology of seed dispersal. Annu. Rev. Ecol. Syst. 13, 201-228.

[9] Lan, S. L. and Sun, M.(2001). Aerodynamic properties of a wing performing unsteady rotational motions at low Reynolds number. Acta Mech. 149, 135-147.

[10] Lentink, D. and Dickinson, M. H. (2009). Rotational accelerations stabilize leading edge vortices on revolving fly wings. J. Exp. Biol. 212, 2705-2719. 